\title{
lactating rats
}

György M. Nagy ${ }^{1,4}$, Pálma Feher², Ibolya Bodnár, Reid Luke' ${ }^{1}$ Zsuzsanna E. Tóth", Miklós Vecsernyés² and Béla E. Tóth²

${ }^{1}$ Ross University School of Medicine - Commonwealth of Dominica, West Indies, ${ }^{2}$ University of Debrecen - Debrecen, Hungary, ${ }^{3}$ EGIS Pharmaceuticals Budapest, Hungary, ${ }^{4}$ Semmelweis University - Budapest, Hungary

\section{Background}

It has been previously shown that hypothalamic control of pituitary adrenocorticotropic hormone (ACTH) secretion switches to dopamine (DA) with a parallel loss of DAerg control of alpha-melanocytestimulating hormone $(\alpha-\mathrm{MSH})$ release in lactating dams (1). It has been also shown that inactivation (dephosphorylation) of tyrosine hydroxylase $(\mathrm{TH})$ in terminals of DA neurons at the median eminence (ME) is required not only for suckling-induced PRL but also for ACTH responses (2).

\section{Aim of the study}

The aim of the present study was to further investigate the regulatory switch of ACTH with special emphasis on potential changes in the expression of D2 DA receptor (D2R) at the level of anterior lobe (AL) and the neuro-intermediate lobes (NIL) of the pituitary gland in lactating rats.

\section{Materials and Methods}

\section{Animals}

Primiparous lactating rats (Sprague-Dawley, 220 $250 \mathrm{~g}$ b.w.) were used at days 7-11 of lactation. Litter size was standardized to 8 from the second postpartum day. Ovariectomy of cycling females was performed 7 days before any experiments. The animals were housed in a temperature-controlled room $\left(21-23^{\circ} \mathrm{C}\right)$ with alternating cycles of $14 \mathrm{~h}$ light and $10 \mathrm{~h}$ darkness. Standard rat chow and water was available ad libitum.

Primary cell culture

The pituitary were removed and AL and IL were separated. Tissue fragments of the AL and IL were dispersed separately with trypsin, aliquoted into 10 $\mathrm{mm}$ Falcon sterile dishes $(\sim 300000$ cells/well of AL or IL), then cultured in DMEM- $0.1 \%$ BSA containing $2.5 \%$ fetal calf serum for $24 \mathrm{~h}$. On the day of experiments, cells were incubated with $1 \mathrm{ml}$ test medium (controls), or exposed to the DA (10-7 M) treatment for 1 hour (3). Aliquots of the supernatants were removed for determination of PRL, ACTH and alpha-MSH.

Radioimmunoassay (RIA)

PRL was measured by using the materials kindly provided by the National Hormone and Pituitary Program (Rockville, Md., USA), $\alpha$-MSH and ACTH were determined by specific radioimmunoassays (RIAs) (4). For measurement of $\alpha-\mathrm{MSH}$, proteins were denaturated by adding $96 \%$ of ethanol to each sample then centrifuged. The supernatants were partially evaporated under N2 environment. The antiserum used in the RIA revealed cross-reactivity as follows: ACTH1-13-NH2 68\%; ACTH1-24 0.2\%; ACTH1-39-ACTH1-32-ACTH1-16-ACTH1-10ACTH1-8-ACTH4-10-ACTH11-24, $\beta$-END, $\beta$ $\mathrm{LPH}, \gamma 3-\mathrm{MSH}$, and $\mathrm{N}$-terminal fragment of human POMC $<0.01 \%$. Detection limit of the assay was $2-3$ $\mathrm{pg} / \mathrm{sample}$. For ACTH measurement, the same ACTH antibody (No. 8514) was used like in immunohistochemistry. All samples were measured in duplicate and from a particular experiment in the same assay. The intra- and inter-assay coefficients of variations were $<10 \%$

\section{In situ hybridization}

The fragment of the rat $\mathrm{D} 2$ receptor cDNA containing nucleotides $372-1,174$ of the coding sequence (GenBank Acc: X53278.1) subcloned into Bluescript KSII + vector was kindly provided by Éva Mezey (5$6)$. The [35S]UTP-labeled antisense riboprobes were prepared by in vitro transcription (Maxiscript KIT, Ambion, Austin TX, USA), according to the manufacturer's protocol. Perfused-fixed pituitaries were cut into $12 \mu \mathrm{m}$ thick serial sections in a cryostat (Leica Microsystems GmbH, Wetzlar, Germany). The sections were thaw mounted and air-dried at $37^{\circ} \mathrm{C}$ onto positively charged Superfrost Plus slides (Thermo Scientific, Budapest, Hungary). Hybridizations were performed overnight in humid chambers at $55^{\circ} \mathrm{C}$ with $106 \mathrm{cpm} /$ slide of the [35S]UTP-labeled probes, as described earlier (7).

\section{Immunohistochemistry}

After hybridization and washing, sections were rinsed in PBS and processed for ACTH immunohistochemistry, using the $\mathrm{ABC}$ method and $\mathrm{DAB}$ visualization. Then the sections were dehydrated and the slides were dipped into Kodak NTB nuclear track emulsion (Carestream Health Inc., Rochester, NY, USA). In situ hybridization signal was developed after 10 days of incubation at $4^{\circ} \mathrm{C}$ in dark using Kodak Dektol developer and fixer (Sigma-Aldrich Kft. Budapest, Hungary). Finally the sections were air dried and covers placed using DPX mounting medium. (5-7)

Results
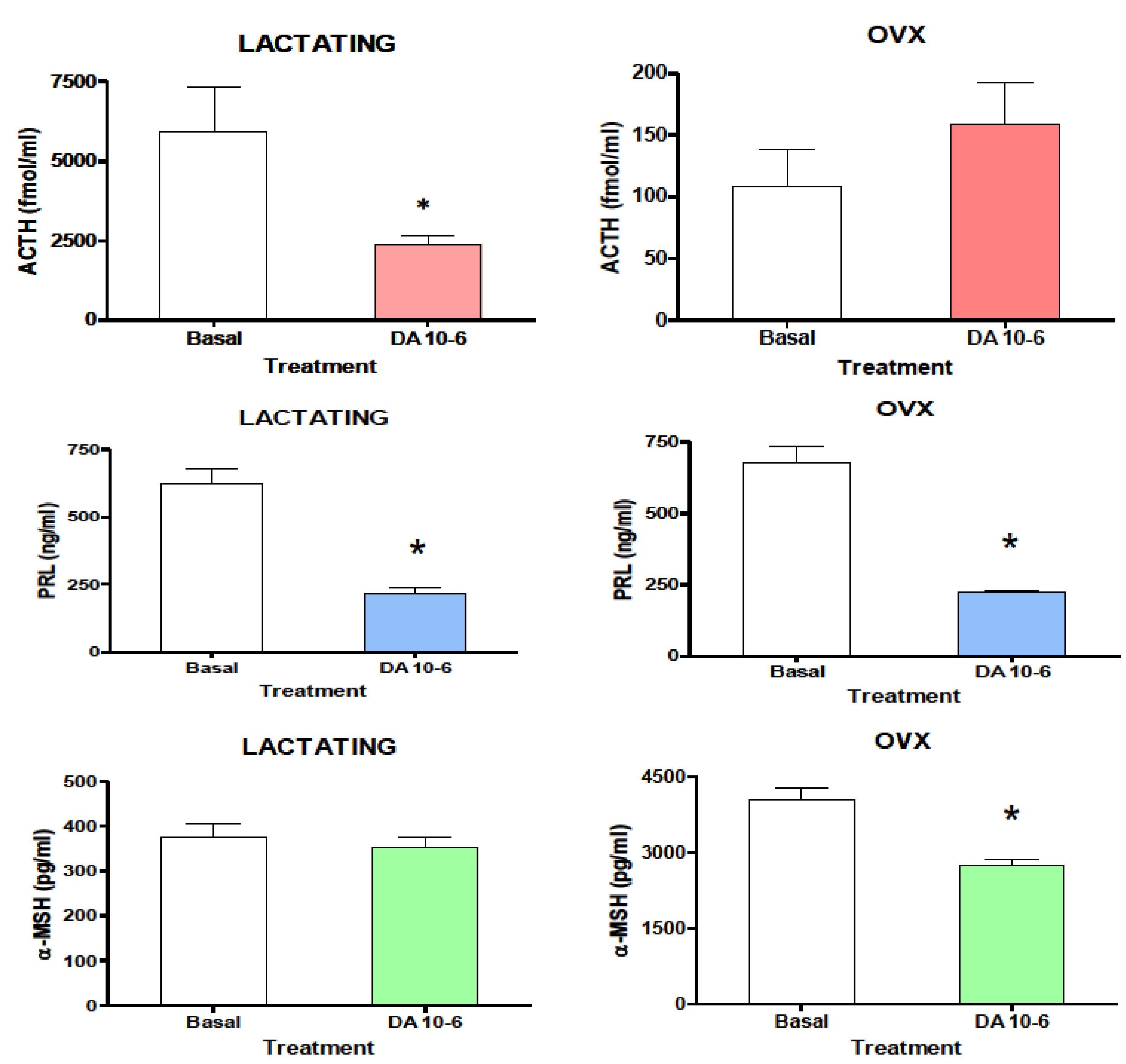

Fig 1. In cells obtained from OVX rats, ACTH secretion was not sensitive to DA, but both PRL and $\alpha$-MSH were blunted. In contrast, on AL cells obtained from lactating rats, DA treatment resulted in an inhibition of both PRL and ACTH secretion but had no effect on $\alpha$-MSH. * $\mathrm{P}<0.05$,

ACTH immunostaining and D2 receptor in situ hybridization in the anterior lobe $(\mathrm{AL})$ of the pituitary gland.

ovx



LACTATING
ACTH immunostaining and $D 2$ receptor in situ hybridization in the anterior lobe (AL) of the pituitary gland.
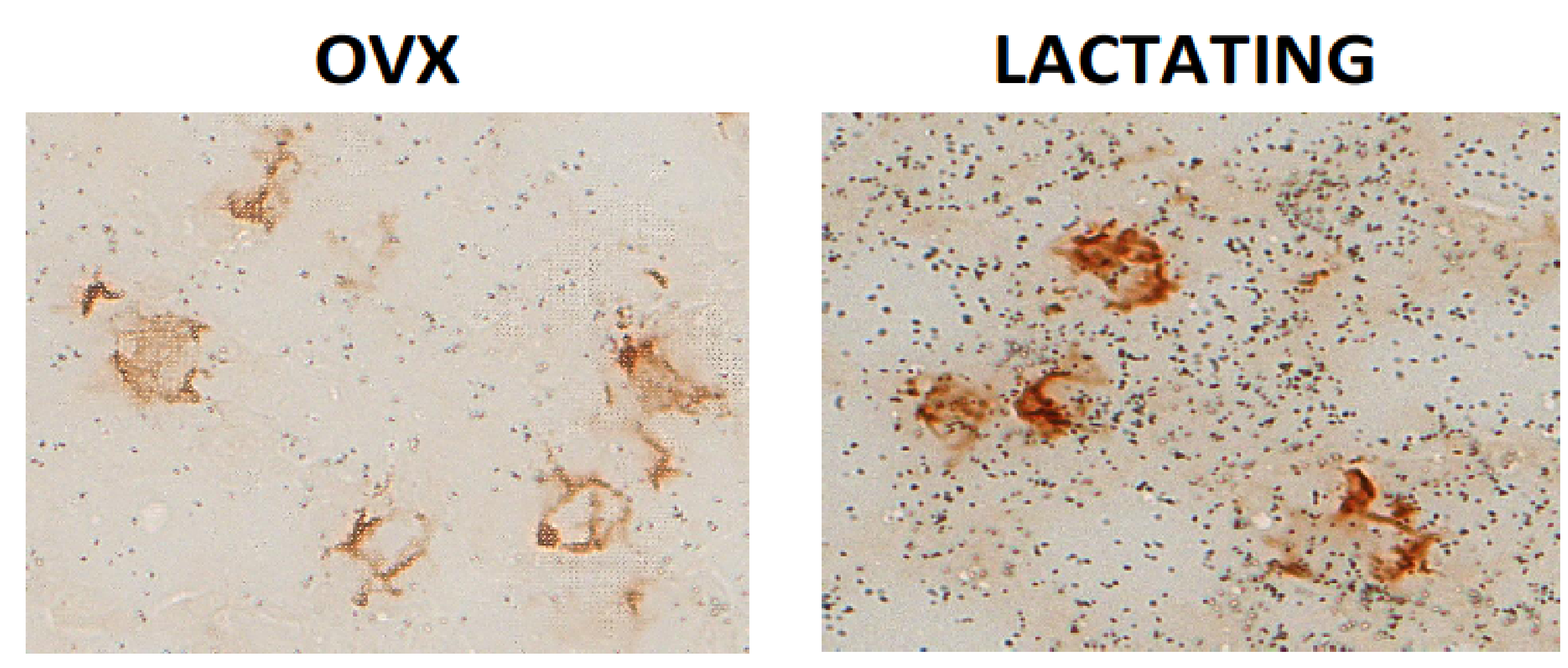

Figure 2(B)

Fig 2 A and B. ACTH immunohistochemistry and D2 DA receptors (D2R) in situ hybridization techniques were combined to investigate whether DA inhibition of ACTH secretion is due to the expression of $\mathrm{D} 2 \mathrm{R}$ on corticotrophs. Expression of D2R was much higher in both the AL and the NIL of lactating dams compared to OVX animals

This study revealed co-localization of D2R with ACTH in a subpopulation of corticotroph cells of lactating animals.

Table I.

Dopamine D2 receptor (D2R) expressions were detected by in situ hybridization method in the anterior lobe (AL) of the pituitary gland in lactating (LACT) and ovariectomized (OVX) rats.

Particle counting (DR2 / ACTH+)

$\begin{array}{llll}\text { OVX AL } & \underline{\text { AVG }} & \underline{\text { SD }} & \underline{\text { SEM }} \\ \text { LACT AL } & \mathbf{3 . 4 9} & \mathbf{1 . 3 7 6} & \mathbf{0 . 4 8 6} \\ & 8.23 * & 0.534 & 0.308\end{array}$

* Indicates statistical significance compaired with OVX AL group $(p<0.05)$. Counting was performed by ImageJ software.

\section{Summary and Conclusions}

Our data show that DA sensitivity of ACTH secretion is most probably due to the expression of D2R in a subpopulation of corticotrophs in lactating animals. These cells may also be responsible for the higher basal activity of the hypothalamo-pituitary-adrenal (HPA) and the stress hyporesponsiveness during lactation. The fact that melanotrophs loose their DA sensitivity in spite of similar increase of D2R expression in the IL (data not shown) indicate a lack of transdifferentiation from melanotrophs to corticotrophs and migration from the AL to the IL It has been already shown that most corticotropinomas express functional D2R (8-10), and ACTH reduction in response to DA is directly associated with D2R expression (8). It is important to note that a switch of DAerg regulation from melanotrophs to corticotrophs can occur during physiological circumstance. It indicates that in the development of a DA sensitive corticotroph adenoma is most likely due to a more complex and integrated event on both at the hypothalamic and the adenohypophyseal level.



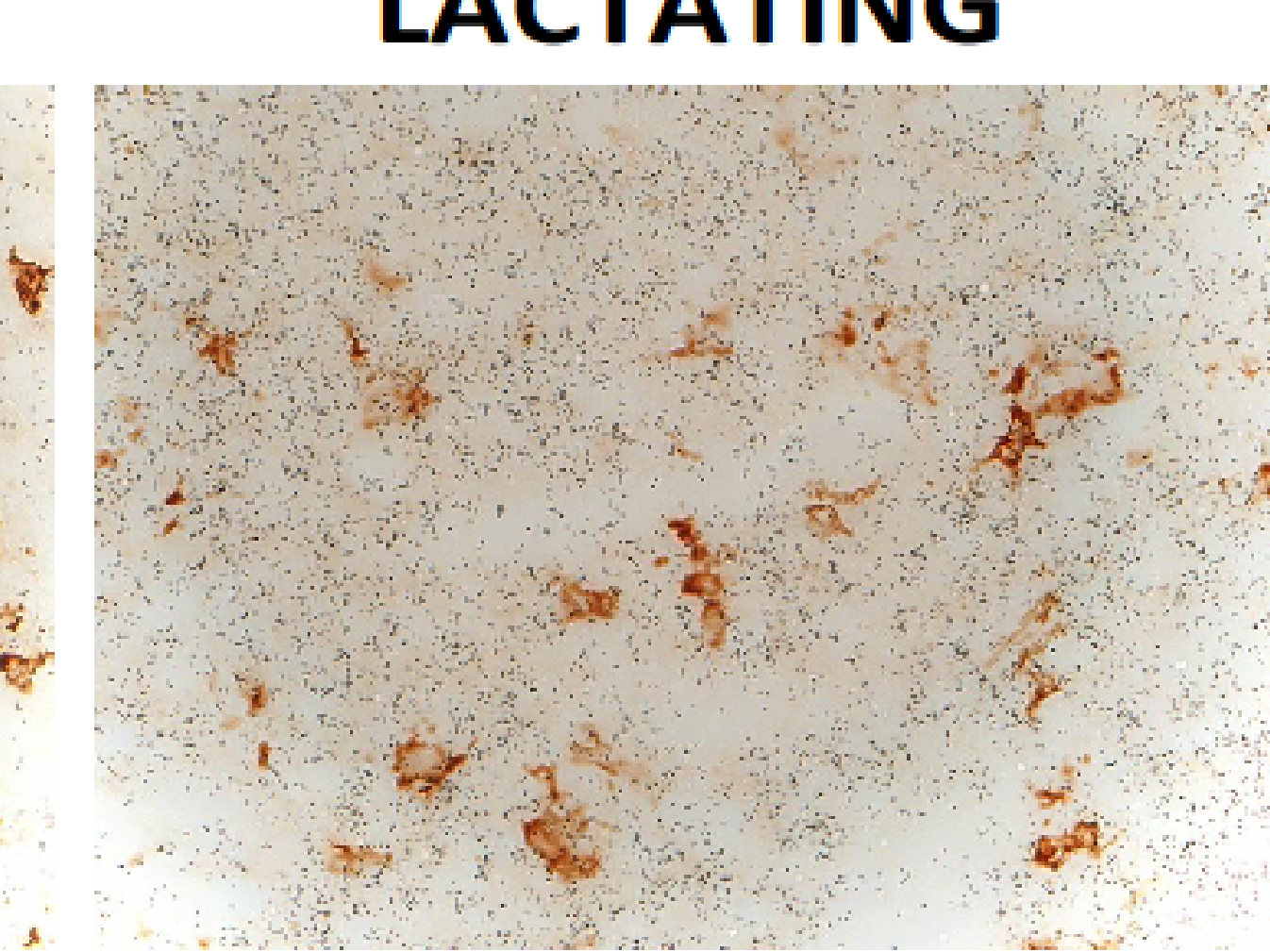

